\title{
Network isolation and local diversity in neutral metacommunities
}

\author{
Evan P. Economo and Timothy H. Keitt \\ E. P. Economo (economo@umich.edu) and T. H. Keitt, Section of Integrative Biology, Univ. of Texas at Austin, 1 University Station A6700, \\ Austin, TX 78712, USA. Present address for EPE: 2004 Kraus Nat. Sci. Bldg., 830 N. University St., Ann Arbor, MI 48109, USA.
}

\begin{abstract}
Biologists seek an understanding of the biological and environmental factors determining local community diversity. Recent advances in metacommunity ecology, and neutral theory in particular, highlight the importance of dispersal processes interacting with the spatial structure of a landscape for generating spatial patterns and maintaining biodiversity. The relative spatial isolation of a community is traditionally thought to have a large influence on local diversity. However, isolation remains an elusive concept to quantify, particularly in metacommunities with complex spatial structure. We represent the metacommunity as a network of local communities, and use network centrality measures to quantify the isolation of a local community. Using spatially explicit neutral theory, we examine how node position predicts variation in alpha diversity across a metacommunity. We find that diversity increases with node centrality in the network, but only when centrality is measured on a given scale in the network that widens with increasing dispersal rates and narrows with increasing evolutionary rates. More generally, complex biodiversity patterns form only when the underlying geography has structure on this critical scale. This provides a framework for understanding the influence of spatial geographic structure on global biodiversity patterns.
\end{abstract}

A fundamental task of basic and applied ecology is to understand what determines local community diversity (Ricklefs 1987, Brown 1995, Rosenzweig 1995). Ecological theory increasingly suggests that local diversity depends on spatial dynamics occurring on larger scales. Many ecological communities are embedded in a metacommunity, a network of local communities linked through dispersal. Recent theory has established the importance of these connections for changing and propagating ecological dynamics (Levin 1992, Hubbell 2001, Holt 2002, Amarasekare 2003, Leibold et al. 2004).

A key variable in most spatial biological theory, including metapopulation (Hanski 1999), biogeographic (MacArthur and Wilson 1967), and metacommunity theory (Hubbell 2001, Leibold et al. 2004) is isolation of a patch or community. However, isolation is a scale dependent concept (Keitt et al. 1997), and the relevant scale for determining local diversity is often unclear. Communities may be isolated relative to their local landscapes, in a regional network of patches, or relative to the rest of their entire biogeographic province. Which scales of isolation control local diversity? And what biological parameters set those scales?

Ecological neutral theory (Hubbell 2001, Chave 2004) provides a quantitative, mechanistic framework for understanding biodiversity patterns in metacommunities in terms of a minimal set of stochastic biological processes. It resides at one extreme in a continuum of metacommunity models that vary in their emphasis on the importance of niche differences, species interactions, and stochastic spatial effects on shaping ecological communities (Leibold et al. 2004, Alonso et al. 2006, Leibold and McPeek 2006). Despite its limitations, in certain cases it has proven surprisingly capable at predicting community patterns found in nature (Hubbell 2001, McGill et al. 2006, Muneepeerakul et al. 2008).

Neutral theory highlights spatial effects due to the central role of dispersal limitation in determining community structure. However, as many of the neutral models explored in the literature are either spatially implicit (Hubbell 2001, Etienne 2005) or constructed with two dimensional landscapes (Durrett and Levin 1996, Bell 2000, Hubbell 2001, Chave and Leigh 2002, Condit et al. 2002, Rosindell and Cornell 2007), the consequences of spatial complexity for neutral pattern are still largely unknown.

Recent work (Muneepeerakul et al. 2007, 2008, Economo and Keitt 2008) has sought to address this limitation by representing neutral metacommunities as networks, with local communities (nodes) connected by the capacity for dispersal (links or edges). Networks are ideally suited for representing a set of units with complex connections among them, and their utility across disciplines (Strogatz 2001) has led to the wide application of a common set of quantitative tools (Newman 2003). In ecology, spatially complex landscapes can be represented as a graph (Keitt et al. 1997, Urban and Keitt 2001, Bodin and Norberg 2007, Economo and Keitt 2008, Estrada and Bodin 2008, Minor and Urban 
2008, Urban et al. 2009). The structure of the graph could reflect, for example, the arrangements of islands in an archipelago or mountains in a range. An advantage of the network framework is a sophisticated set of quantitative tools available for characterization of network structure (Urban and Keitt 2001, Newman 2003). We seek to identify which concepts and tools may be useful to spatially explicit metacommunity theory.

In the spatially implicit neutral model, differences in diversity among local communities are driven by migration rates from the metacommunity and the local community size (Hubbell 2001, Etienne 2005, 2007). Migration rate is commonly measured by $\mathrm{m}$, or the probability an individual originated outside the local community. This general idea can be traced back to classical island biogeography theory, which emphasized the importance of island isolation on alpha diversity (MacArthur and Wilson 1967). In the network model (Economo and Keitt 2008), the migration rate $(\mathrm{m})$ corresponds to node degree, the number of links a community has with other communities (or the sum of their weights). This places an emphasis on the immediate neighborhood of a node on determining alpha diversity.

However, in a network of communities, diversity may not simply be a function of connectivity with neighboring patches as those patches may serve as stepping stones to other regions of the metacommunity. If diversity cascades through the patch network in this manner, local diversity would be driven by metacommunity structure at broader scales than the local neighborhood. This requires a more sophisticated implementation of the concept of isolation.

Here we consider equilibrium alpha diversity at different rates of migration (edge weights) and speciation, in two model networks with different types of complex structure. These are a dendritic graph with a tree-like branching topology, and a modular graph with connected clusters of nodes. The dendritic graph allows for relatively easy visualization of node position in the network, and increases variation in topological distance compared to more complex topologies. The modular graph is intended to echo the complexity arising from hierarchically patchy landscapes. To further focus the problem, we only consider symmetric networks, where migration is equal in both directions between two communities.

We use 'network centrality' measures to quantify the position of a node in a metacommunity. These statistics, originally developed by the social sciences (Freeman 1978, Wasserman and Faust 1994, Scott 2000), and are used across disciplines to quantify network topology. We use them as different quantitative implementations of the concept of patch isolation in spatial ecology and biogeography. Centrality measures vary in their emphasis on short range or long range connections and how paths between nodes are interpreted. Such measures have been used before to quantify local and regional node position in landscape networks (Estrada and Bodin 2008). Establishing which of these predict neutral diversity, and under what biological conditions, reveals fundamental properties of how spatial structure drives neutral diversity pattern in complex metacommunities. It also represents the first implementation of network isolation concepts for metacommunities that are applicable well beyond neutral theory.

\section{Methods}

\section{A network concept of isolation in a metacommunity}

We used a variety network centrality measures to quantify the position of a node in a metacommunity with network structure. Node isolation is the inverse of node centrality. Each measure highlights a different aspect of network topology. 1) 'Degree centrality' is simply the number of links a node has with other nodes or the sum of their weights. In a landscape, this corresponds to the flux of dispersal directly entering a node from other nodes. 2) The 'geodesic closeness centrality' (Wasserman and Faust 1994, Scott 2000) is the average shortest path length between a node and all other nodes in the network. This measures the position of a node in the broader landscape, literally how close a node is to all other nodes. Note that a node with high closeness can have very low degree, and vice versa. 3) The 'resistance closeness centrality' is identical to geodesic closeness except the resistance distance (McRae 2006) takes the place of geodesic distance. The resistance distance, which is derived from circuit theory, shortens when there are multiple paths between nodes while geodesic distance is solely determined by the shortest path. 4) 'Eigenvector centrality' (Bonacich 1972) scores nodes based not only by the number of connections with neighbors, but also by the importance of their neighbors. The metrics thus far all have a particular spatial scope, with degree centrality a function of local structure and the rest a function of structure of the entire network. As a final metric, it is desirable to have a statistic that can be tuned to reflect structure on intermediate scales. 5) We thus introduce the 'k-neighborhood' - the number of other nodes that are within a geodesic distance $\mathrm{k}$ from the focal node. The degree centrality is the size of the $\mathrm{k}$-neighborhood when $\mathrm{k}=1$.

As measures of global position of a node in the network, we favored measures of closeness centrality over betweenness centrality (Wasserman and Faust 1994, Scott 2000). Betweenness centrality considers how often a node is traversed on either shortest paths or random walks between other nodes, which are important in cases where one may need to identify key connecters in networks of information flow. To understand why we would favor closeness centrality, consider a node that is near the geographical center of the network but has only one link connecting itself to the network (a dead end). Such a node would have short path lengths to many other nodes - a high closeness centrality but would never be intermediate on paths between another pair of nodes. Thus the node would have the lowest possible betweenness score, the same betweenness as a truly peripheral node in the most isolated regions of the network. This makes it a non-ideal measure of the topological position only with regards to the processes of interest in this paper. Here we seek to understand how the global position in the network, which should differ between a dead end link near the center of the network and a dead end in a peripheral region, affects diversity.

The centrality measures were calculated on the adjacency matrix. Degree centrality is simply the number of links connected to a node. Closeness centrality is generally a measure of the position of a node relative to the rest of the 
network, usually calculated as the average distance between a node and all other nodes. We calculate two variants of closeness centrality based on two measures of network distance (Wasserman and Faust 1994, Scott 2000). Geodesic closeness centrality is based on geodesic distances (shortest path lengths) which we found using Dijkstra's algorithm. Resistance closeness centrality uses the resistance distance, which we calculated using the method described by McRae (2006). Resistance distance and geodesic distance are equivalent for tree-like graphs, such as the dendritic network used in this paper. The closeness measure for node $\mathrm{i}$ was then simply the inverse of the mean distance between $i$ and all $j$, using either distance metric. Eigenvector centrality is the eigenvector associated with the largest eigenvalue of the adjacency matrix, and essentially ranks nodes not only by their immediate connections but the degree of those connections. The size of the $\mathrm{k}$-neighborhood of node $\mathrm{i}$ is the number of nodes within a geodesic distance $\mathrm{k}$ of the focal node.

\section{Network construction}

We built two networks with complex spatial structure, one with a tree like, dendritic topology (Fig. 1) and one with modular structure (Fig. 2). As the focus of our paper is how the position of a node in a metacommunity affects diversity, we avoided networks that had overly symmetric or entirely random topologies, as in both of those cases, node position tends to be similar across the network. The dendritic network was assembled by randomly connecting nodes, but with the constraints that the 200 nodes should have 199 bidirectional links, and it should be one connected component (all nodes must be reachable on a path from all other nodes). We created the modular network by generating subnetworks with random topologies, then haphazardly connecting those (connectance $=0.022$ ). The property featured in the latter network is sometimes called community structure (Girvan and Newman 2002) in the network literature, but to avoid confusion with the concept of biological community structure, we use the 'modular' label which is also familiar to ecologists (Olesen et al. 2007).

\section{Neutral diversity theory}

The neutral model we examine here assumes a constant metacommunity and local community size. In each generation, an individual is drawn randomly from a parent in the same community with probability $m_{i i}$, from another node $j$ with probability $\mathrm{m}_{\mathrm{ij}}$, and is a new species with probability $\mathrm{v}$. We refer to $\mathrm{v}$ as the speciation rate, but it also could represent immigration from a large source pool. The set of all $\mathrm{m}_{\mathrm{ij}}$, is the migration matrix $\mathbf{M}$, and describes the structure of the metacommunity. For the purposes of this paper, all migration rates (links) in the metacommunity are set to the same value and all community sizes $\mathrm{N}_{i}$ are equal.

We used the quantitative method developed in a previous paper (Economo and Keitt 2008), for calculating expected neutral diversity in a network of communities under these assumptions. The method adapts equations originally developed from neutral population genetics theory. The quantity we wish to calculate is the probability of identity in state $f_{i j}$ of two individuals chosen from communities $i$ and $j$ (i.e. the probability the two individuals are the same species). This probability can then be converted to diversity statistics such as Simpson's index of diversity $1-\mathrm{f}_{\mathrm{ii}}$ (Simpson 1949). In this paper, our measure of alpha-diversity is the Simpson concentration $\left(f_{\mathrm{ii}}\right)$ converted to an effective species richness, $\mathrm{f}_{\mathrm{ii}}^{-1}$ that does not converge as diversity becomes large (this is also sometimes called Simpson's index or Simpson's reciprocal index) (Hill 1973). Probability of identity can be calculated with the following recursive equation (Economo and Keitt 2008):

$$
\begin{aligned}
\mathrm{f}_{\mathrm{ij}}= & (1-\mathrm{v})^{2}\left[\sum_{\mathrm{k}, \mathrm{l}, \mathrm{k} \neq 1} \mathrm{~m}_{\mathrm{ik}} \mathrm{m}_{\mathrm{j} 1} \mathrm{f}_{\mathrm{kl}}+\sum_{\mathrm{k}} \mathrm{m}_{\mathrm{ik}} \mathrm{m}_{\mathrm{jk}}\left(1-\mathrm{N}_{\mathrm{k}}^{-1}\right)\left(\mathrm{f}_{\mathrm{kk}}\right)\right. \\
& \left.+\sum_{\mathrm{k}} \mathrm{m}_{\mathrm{ik}} \mathrm{m}_{\mathrm{jk}} \mathrm{N}_{\mathrm{k}}^{-1}\right]
\end{aligned}
$$

Two sampled individuals are the same type if neither has speciated since the previous generation (the first term), and 1) they were from parents of the same type from different patches (the first summation) or 2) they were from different parents of the same type located in the same patch (second summation), or 3) they had the same parent (coalesced) in the previous generation (third summation). For a network of $\mathrm{n}$ nodes, there $\operatorname{are} \mathrm{n}^{2}(\mathrm{i}, \mathrm{j})$ pairs, and thus $\mathrm{n}^{2}$ linear equations in this form describe the system at equilibrium. Since there are $\mathrm{n}^{2}$ unknowns in $\mathrm{n}^{2}$ equations, the system can be solved for the vector $\vec{f}$ of all $f_{i j}$. For the analyses in this paper, used Eq. 1 to construct an $\mathrm{n}^{2} \mathrm{x} \mathrm{n}^{2}$ matrix $\mathbf{X}$, and the right side as a vector $\overrightarrow{\mathrm{q}}$ of length $\mathrm{n}^{2}$, where:

$$
\mathrm{X}_{(\mathrm{i}),(\mathrm{kl})}=(1-\mathrm{v})^{-2} \delta_{(\mathrm{ij}),(\mathrm{kl})}-\mathrm{m}_{\mathrm{ik}} \mathrm{m}_{\mathrm{jl}}+\delta_{\mathrm{k}, \mathrm{l}} \mathrm{m}_{\mathrm{ik}} \mathrm{m}_{\mathrm{jk}} \mathrm{N}_{\mathrm{k}}^{-1}
$$

and

$\mathrm{q}_{\mathrm{ij}}=\sum_{\mathrm{k}} \mathrm{m}_{\mathrm{ik}} \mathrm{m}_{\mathrm{jk}} \mathrm{N}_{\mathrm{k}}^{-1}$

and $\delta_{i, j}$ is the Kroenecker delta $\left(\delta_{i, j}=1\right.$ only when $i=j$ and is 0 otherwise). The formula $X \vec{f}=\vec{q}$ can then be solved for the vector $\vec{f}$ of probability of identities.

\section{Results}

Figure $1 \mathrm{a}-\mathrm{b}$ represents the degree and geodesic closeness centrality on the dendritic network, and Fig. 2 plots resistance closeness centrality and degree centrality on the modular network. Notice that degree centrality is dependent on the local neighborhood of a node, while geodesic closeness integrates the position of the node in the entire metacommunity. Similar plots for both networks and all centrality statistics are presented in Supplemental material Appendix 1 Fig. S1.

Figure 1b-d plots alpha diversity of each node at different rates of migration. As migration rate increases, alpha diversity increases for all nodes at the expense of beta diversity, as was demonstrated in a previous analysis (Economo and Keitt 


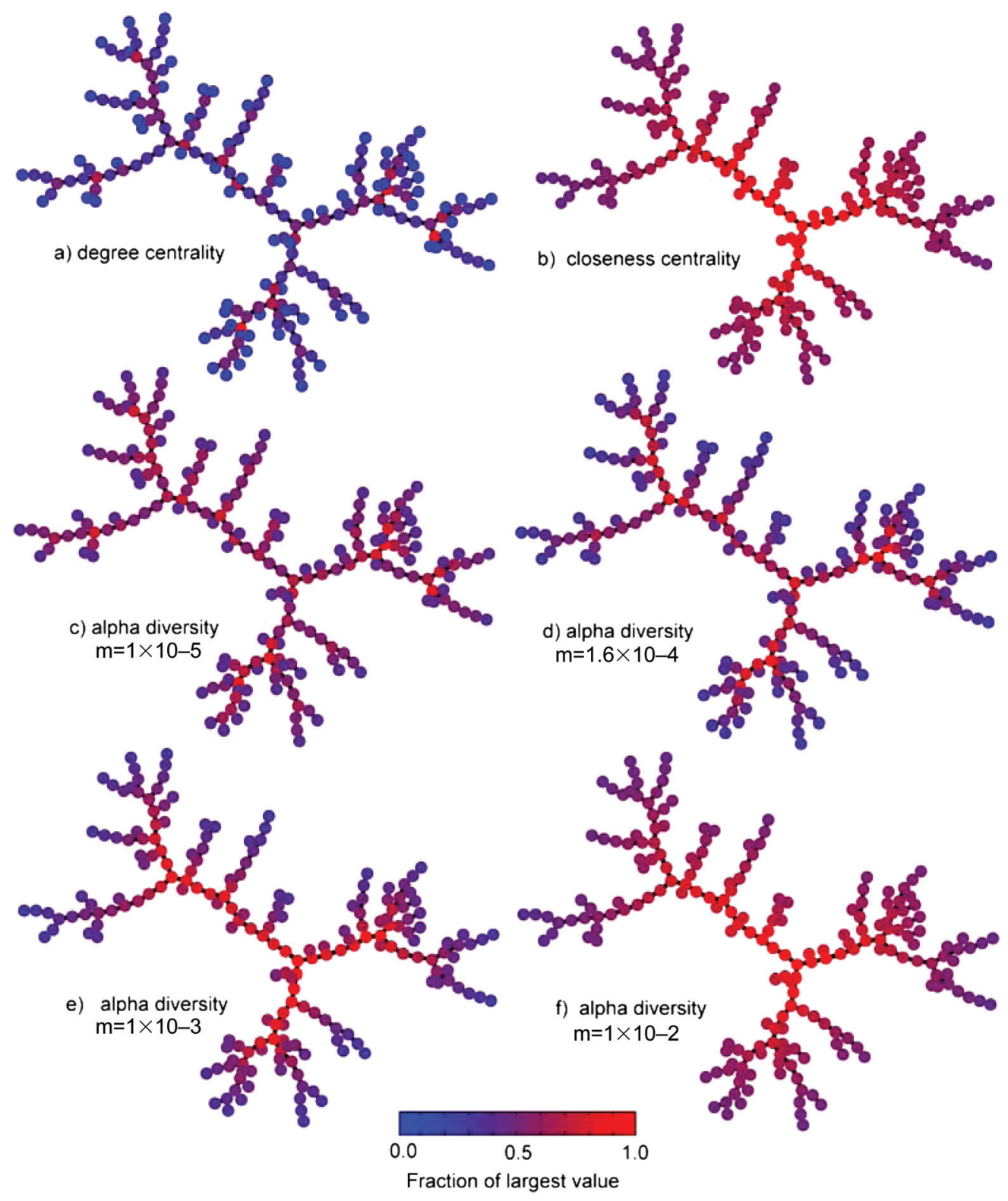

Figure 1. Visualization of centrality and diversity patterns in a metacommunity with network structure. Node degree (a) and closeness centrality (b), and equilibrium alpha diversity at various migration rates (c-f), across the dendritic network. For the latter, $v=10^{-5}$ and $\mathrm{N}_{\mathrm{k}}=200000$.

2008). In each panel, color variation was normalized to the most diverse community. This allows us to visually examine the variation in diversity across the network and across parameter values, which are presented in both Fig. 1c-f and Supplemental material Appendix 1 Fig. S2. When dispersal is low, (Fig. 1c), it is apparent that node diversity (color) is only a function of the local connectivity, nodes near the center of the network, but only connected to one other node, have a similar diversity to a node that has one connection but is on the periphery of the node. The diversity pattern closely resembles the pattern created by degree centrality (Fig. 1a). As migration is increased by an order of magnitude (Fig. 1d), the most diverse nodes are now not simply those with high degree, but those occurring at a confluence of branches, making them within a few steps of a larger number of nodes than those near the periphery. As migration is increased further (Fig. 1e), the most diverse nodes are those that connect large branches, or major divisions in the spatial structure of the metacommunity. Finally, when migration is highest, the most diverse nodes are those most central to the entire metacommunity (Fig. 1f).

We examined the correlations of network centrality measures with node position, which should quantify the transition observed in the visual patterns of Fig. 1. In Fig. 3, the alpha diversity of each node in the dendritic and modular networks, is plotted as a function of three different measures of node centrality and four different migration rates. For resistance closeness centrality (Fig. 3a, 3d), which integrates the position of the node in the entire network, correlations with alpha diversity are stronger when migration rates are 


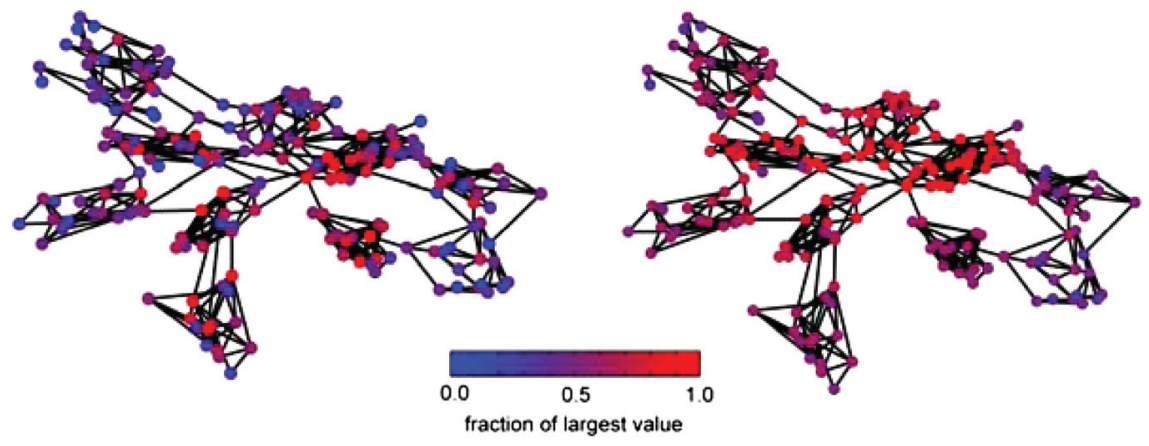

Degree centrality

Resistance closeness centrality

Figure 2. The modular network used in this paper with nodes colored by degree centrality and resistance closeness centrality.

high, and lower when migration rates are low. Degree centrality (Fig. 3b, 3d), a local measure, is strongly correlated at low migration rates but increasingly becomes less correlated at higher migration rates. Eigenvector centrality is relatively weakly correlated with diversity at any migration rate. This appears to be because eigenvector centrality picks out the most highly connected cluster and assigns nodes within it a higher score than even nodes that have similar connectivity in another section of the network (Supplementary material Appendix 1 Fig. S1d).

These correlations are presented in Fig. 4, showing the tradeoffs of local versus network wide controls on alpha diversity. The centrality metrics are not uncorrelated with each other, having a high degree also decreases distance to other regions of the network (particularly in relatively small networks such as these).
At extremes of low and high migration, which can be seen in Supplementary material Appendix 1 Fig. S2, alpha diversity is nearly constant across the metacommunity. This is because each node becomes an independent community (when migration is low) or the metacommunity is effectively panmictic (at high migration). At high migration rates, and spatial position of a node has little effect on diversity patterns, alpha diversity is constant across the metacommunity. At the other extreme, when migration is very restricted, each individual node becomes a unique community, and again alpha diversity is basically constant across the metacommunity. Variation in alpha diversity, a proxy for the complexity of spatial pattern, peaks at intermediate migration (Fig. 4c-d).

It is clear in Fig. 4c-d, that variation peak shifts at different speciation rates. This implies that, given a physical

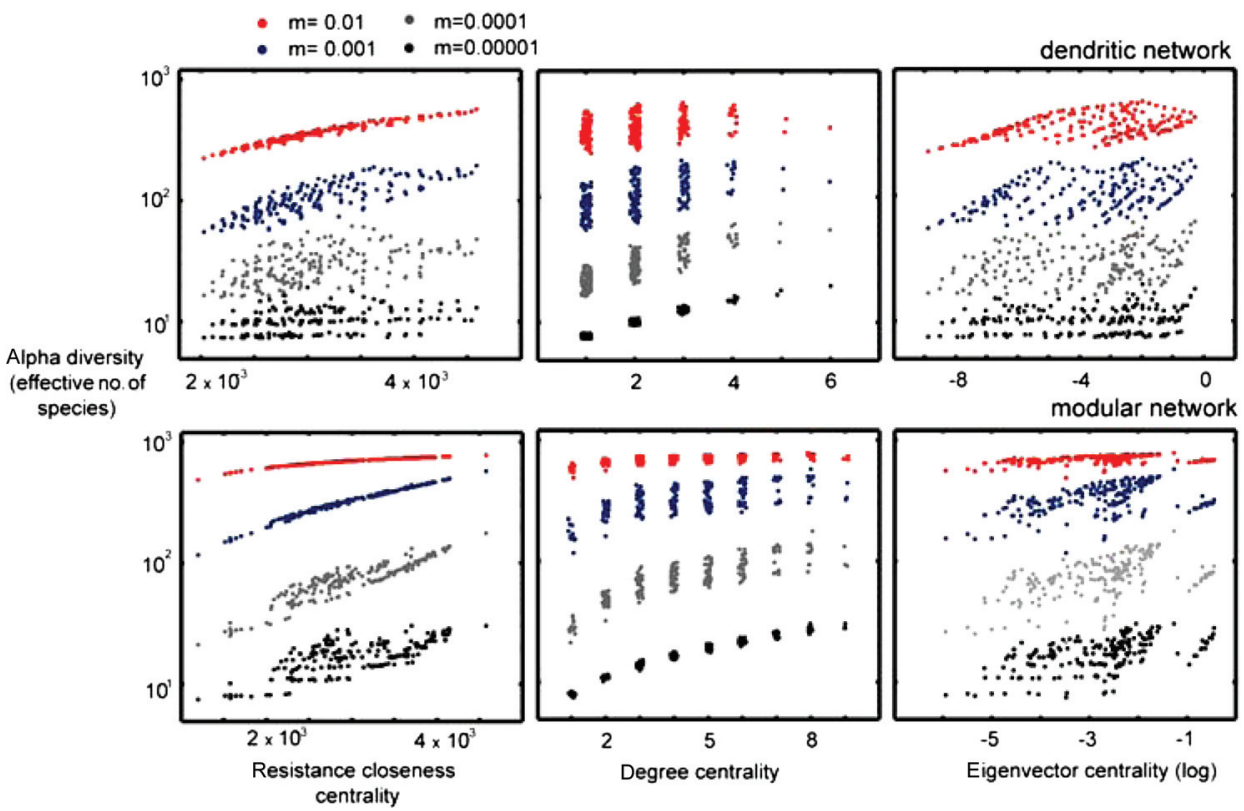

Figure 3. Alpha diversity of local communities (nodes) plotted as a function of three centrality metrics for the dendritic and modular networks, at different rates of migration. As migration rates increase, network-scale centrality measures (resistance closeness) better predict diversity, while smaller scale metrics (degree centrality) predicts diversity variation better under restricted migration. Eigenvector centrality only weakly predicts alpha diversity. Speciation rate and local community size were held constant, $\mathrm{v}=10^{-5}, \mathrm{~N}_{\mathrm{k}}=200000$. 


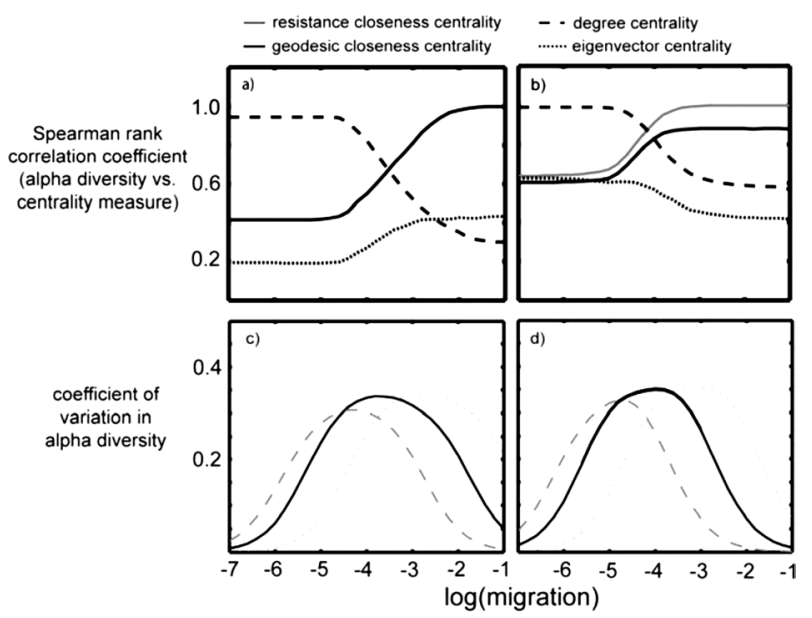

Figure 4. Performance of different centrality statistics across parameter values. Spearman rank correlation coefficients of node alpha diversity and network centrality metrics are plotted for the (a) dendritic and (b) modular network. The geodesic and resistance closeness centralities are equivalent for the dendritic network. Variation in alpha diversity across the network, as measured by coefficient of variation, is presented in $\mathrm{c}-\mathrm{d}$. For a given speciation rate, variation peaks at an intermediate migration rate. The black line reflects a speciation rate of $\mathrm{v}=10^{-5}$ corresponding to the correlations in $(\mathrm{a}-\mathrm{b})$, the grey dashed line is $\mathrm{v}=10^{-6}$, and grey dotted line is $\mathrm{v}=$ $10^{-4}$. Thus, for a given topology, variation in alpha diversity depends strongly on the relative rate of speciation and migration.

landscape structure, there is a particular combination of migration and speciation rates that produces complex spatial variation in alpha diversity. Or, put another way, for a given taxonomic group with certain propensities for dispersal and evolution, they will form complex biodiversity patterns and respond to geographic features only when there is landscape structure on a particular spatial scale.

Degree and closeness centrality measure node position relative to the local and global structure of the network, respectively, with each predicting alpha diversity when migration is high or low. We use the size of the k-neighborhood as a metric that measures spatial structure as scale is increased. This metric is the number of unique nodes within $\mathrm{k}$ steps of the focal node (degree centrality is k-neighborhood when $\mathrm{k}=1$ ). Figure 5 plots the correlation of alpha diversity with the size of the $\mathrm{k}$-neighborhood. When migration is low, $\mathrm{k}=1$ has the most predictive power. As migration increases the most relevant value of $\mathrm{k}$, which is a surrogate for spatial scale, increases.

\section{Discussion}

These results illustrate both the promise and challenges for a network concept of isolation in metacommunities. The centrality measures capture different aspects of network structure, all of which are likely relevant to metacommunity dynamics and should be useful for a wide range of studies. In the neutral scenario considered here, the general idea that more isolated areas have depressed diversity in a neutral metacommunity is supported. However, it is clear that no single quantitative definition of isolation universally predicts

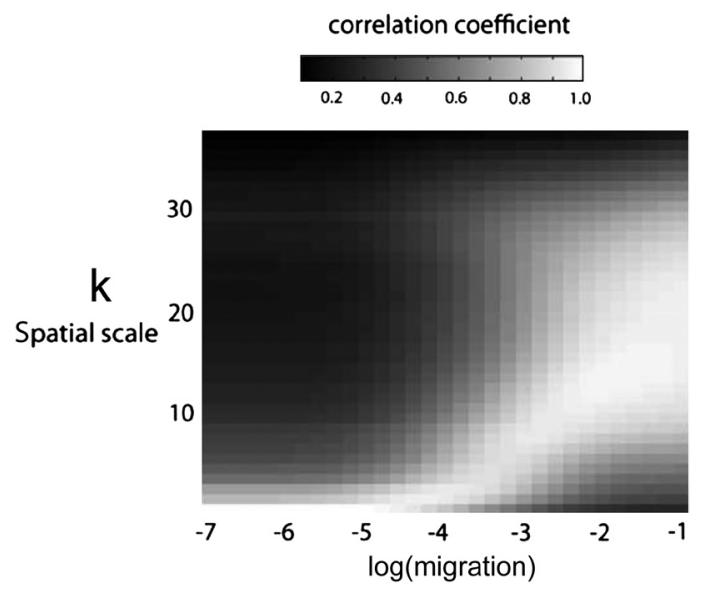

Figure 5. Performance of scale-specific centrality. Spearman rank correlation coefficient of alpha diversity and the size of the kneighborhood at different migration rates and values of $\mathrm{k}$. Larger $\mathrm{k}$ reflect structure on larger spatial scales, and better predict diversity at higher migration rates. Speciation rate and local community sizes were held constant, $\mathrm{v}=0.00001, \mathrm{~N}_{\mathrm{k}}=200000$.

this variation. Rather, isolation predicts diversity only when measured on a critical scale in the network, which is scaled by the relative rates of dispersal and evolution. When migration is more restricted, isolation depresses diversity only when calculated locally. As migration increases relative to speciation, isolation best predicts diversity when calculated at successively greater scales in the network.

This implies that for a given taxonomic group with particular propensities for dispersal and speciation, correlations between isolation and alpha diversity should only be observed at certain spatial scales. This may explain why the isolation component of the island biogeography effect, which is usually arbitrarily defined as a distance to another land mass, is only sometimes observed. In general, more dispersive groups should show a correlation between isolation and alpha diversity on greater spatial scales. For example, the fine scale arrangement of islands in an archipelago would have little effect on diversity variation in a highly dispersive group, which would be driven more by the position of the archipelago in a broader network of archipelagoes rather than isolation within the archipelago. For a more sedentary group, only the fine scale structure of an archipelago, and not the broader regional context, would set diversity of an island. More generally, for complex biodiversity patterns to form in a landscape due to neutral processes, the geography must have structure on this critical scale.

These results have relevance to several other well-known biogeographic phenomena. Geometric effects on diversity patterns have been much discussed. These include the peninsular effect (Simpson 1964), which proposes a decrease in diversity towards the terminal end of peninsulas. This is commonly explained by reduced colonization rates due to the geometry of the underlying spatial template. Neutral theory also predicts a peninsular effect, but only if migration and speciation rates are in the right balance to reflect that geographic feature. Mid-domain effects (Colwell and Lees 2000) predict diversity to peak in the middle of a domain, a pattern that is sometimes - but not always exhibited, and explanations 
have been offered by a number of mechanisms including stochastic neutral effects (Rangel and Diniz-Filho 2005). This can be generalized to a network, where a network-wide most central node takes the place of geographic center of a domain. Our results suggest that the mid-domain effect does not always hold under neutrality in a spatially complex landscape, as migration becomes more restricted, regional spatial structure overrides the broader metacommunity geometry; so mid-domain effects may be more localized or not present at all. However, the basic conclusion that neutral theory can cause mid-domain effects in some regions of parameter space is supported, and also is in accord with results from population genetics (Wilkins and Wakeley 2002).

These results also highlight how neutral processes can produce elevated diversity in nodes that connect different regions of the network. In a dendritic network (Fig. 1), for certain migration values, the nodes that connected two branches had elevated diversity. These offer a neutral hypothesis for biogeographic mixing zones (Spector 2002), instead of an overlap in environments, certain transitional areas may occur due to geometric effects, those patches that connect disparate portions of the network will receive a mix of species from the two areas.

Our understanding of ecological neutral theory is overall still influenced largely by the behavior of spatially implicit models, but this spatially explicit model provides different answers to some basic properties of neutral diversity patterns. The migration parameter $\mathrm{m}$, defined as the fraction of individuals in a local community that originated in another patch, along with the local community size $\mathrm{N}_{\mathrm{k}}$, are often considered to be the two main parameters setting local diversity. The spatially explicit model considered here clearly demonstrates that in many cases local diversity is set not by the number of individuals migrating into that patch, but by its position in a larger neighborhood of patches, or if migration is strong enough, the entire metacommunity. This spatial diversity cascade occurs at regions of higher migration, the local $\mathrm{m}$ value is swamped by the flow of diversity through the metacommunity.

\section{A reverse-time perspective}

Neutral dynamics can be viewed from equally valid forward or reverse-time (coalescent, Kingman 1982, Rosindell et al. 2008) perspectives. In this case of our results, some understanding can be gained by considering the latter. Our model predicts diversity in terms of probability of identities, the probability that two individuals randomly chosen will be the same type. Conceptually, we are tracing ancestry of two individuals backwards in time and ask if they coalesce before either lineage has speciated. For the case of alpha diversity, we choose two individuals from the same spatial location. This depends on how quickly the two lineages move (via random walks) away from each other in backwards time, because once they become spatially separated they must again enter the same locality before they coalesce. Individuals sampled from nodes that have few connections to other nodes (low degree), or in a region of the network that is not highly connected to the rest of the network, such as a peninsular chain of nodes, will more likely be found in the localized region of the network at more distant times in the past, thus have more opportunity to coalesce before speciation. Increasing migration rate increases the spatial scale that the lineages are likely be found at any time in the past, reducing overall the probability of coalescence (and thus increasing alpha diversity) before speciation, but also making that diversity dependent on the structure of the network in that neighborhood. Thus, at very low migration rates, two lineages are likely to either coalesce or speciate before leaving the node, making spatial location of the node relative to the metacommunity irrelevant. At intermediate migration, lineages may travel some distance before speciation, but will not reach distant portions of the network, so the probability of coalescence is dependent on the structure of the limited local neighborhood of the network. At high migration, the lineages are more likely to explore distant regions of the networks before speciation or coalescence, and so its position in the broader network becomes important. Finally, when migration is very high relative to speciation, the time scale is so long that the initial spatial position of the individuals becomes irrelevant for the long-term probability that the two will coalesce, and diversity is constant across nodes.

\section{Caveats and future directions}

The speciation model used here assumes new species arise as a single individual, with equal probability across all individuals. Thus diversity is constantly being added to the metacommunity at all points equally, the patterns are generated by the subsequent flow of that diversity. Note that aside from speciation, this introduction of novelty could be interpreted as migration from a distant, large, source pool. As a model of speciation, there are undoubtedly cases where this is reasonable, such as such as speciation by polyploidy in plants. However, there are probably many cases where spatial structure is important to the process of speciation itself. This implies an additional complexity -speciation rates, not only migration, may depend on spatial location. Future work, building upon recent neutral models (Hubbell 2005, Etienne et al. 2007, Mouillot and Gaston 2007, Aguiar et al. 2009), is needed to explore this issue and will likely require a different mathematical approach.

The current analytical method is limited to relatively small networks (on the order of 200 nodes for sparse networks, much less for highly connected networks on a normal personal computer). One reason for considering network statistics, which can generally be computed easily on much larger networks, is that they may serve as a surrogate for predicting neutral diversity patterns. We find that they do, but that their performance depends on the parameters of the neutral model. It would be interesting if network statistics were developed that could be used to roughly predict diversity patterns from topology, with migration and speciation rates as parameters, without resorting to the full analytical method.

Here we focused on networks with a fixed topology and varied the strengths of the connections among nodes. This was intentional to highlight the interaction between these two aspects of spatial metacommunity structure. However, if we are comparing two different groups of species with different dispersal rates on the same physical landscape, it 
is possible that both topology and link weights would be different, because increased dispersal rate could result in longer range direct connections among nodes and a more connected topology. Of course, this would also depend on the nature of the intervening matrix and its interaction with the species life histories. To address this issue, an interesting future direction would be to consider how the physical structure of landscapes determines different metacommunity network structures when some functional model of dispersal is applied, and how that changing structure results in different diversity patterns.

Our results show that network structure is a strong determinant of local diversity, and that network tools predict that relationship. This is an important step towards a rigorous understanding of the connection between isolation and biodiversity patterns in complex metacommunities. This study also raises important questions about how metacommunity topology affects diversity processes under different models of ecological dynamics than the one considered here. Incorporating spatially explicit model structures into metacommunity theory, which are more realistic depictions of natural landscapes, is likely to change the outcomes of a wide range of metacommunity dynamics. The question is not a trivial one as understanding the processes driving biodiversity pattern is critical for designing effective strategies to maintain it.

Acknowledgements - EPE acknowledges the financial support of a NSF IGERT Fellowship and a NSF Graduate Research Fellowship.

\section{References}

Aguiar et al. 2009. Global patterns of speciation and diversity. Nature 460: 384-387.

Alonso, D. et al. 2006. The merits of neutral theory. - Trends Ecol. Evol. 21: 451-457.

Amarasekare, P. 2003. Competitive coexistence in spatially structured environments: a synthesis. - Ecol. Lett. 6: 1109-1122.

Bell, G. 2000. The distribution of abundance in neutral communities. - Am. Nat. 155: 606-617.

Bodin, Ö. and Norberg, J. 2007. A network approach for analyzing spatially structured populations in fragmented landscape. Landscape Ecol. 22: 31-44.

Bonacich, P. 1972. Factoring and weighting approach to clique identification. - J. Math. Sociol. 2: 113-120.

Brown, J. H. 1995. Macroecology. - Univ. of Chicago Press.

Chave, J. 2004. Neutral theory and community ecology. - Ecol. Lett. 7: 241-253.

Chave, J. and Leigh Jr, E. G. 2002. A spatially explicit neutral model of beta-diversity in tropical forests. - Theor. Popul. Biol. 62: 153-168.

Colwell, R. K. and Lees, D. C. 2000. The mid-domain effect: geometric constraints on the geography of species richness. Trends Ecol. Evol. 15: 70-76.

Condit, R. et al. 2002. Beta-diversity in tropical forest trees. Science 295: 666-669.

Durrett, R. and Levin, S. 1996. Spatial models for species-area curves. - J. Theor. Biol. 179: 119-127.

Economo, E. and Keitt, T. 2008. Species diversity in neutral metacommunities: a network approach. - Ecol. Lett. 11: 52-62.

Estrada, E. and Bodin, Ö. 2008. Using network centrality measures to manage landscape connectivity. - Ecol. Appl. 18: 1810-1825.

Etienne, R. 2005. A new sampling formula for neutral biodiversity. Ecol. Lett. 8: 253-260.
Etienne, R. 2007. A neutral sampling formula for multiple samples and an exact test of neutrality. - Ecol. Lett. 10: 608-618.

Etienne, R. et al. 2007. Modes of speciation and the neutral theory of biodiversity. - Oikos 116: 241-258.

Freeman, L. 1978. Centrality in social networks. Conceptual clarification. - Social Networks 1: 215-239.

Girvan, M. and Newman, M. 2002. Community structure in social and biological networks. - Proc. Natl Acad. Sci. USA 99: 7821-7826.

Hanski, I. 1999. Metapopulation ecology. - Oxford Univ. Press.

Hill, M. 1973. Diversity and evenness: a unifying notation and its consequences. - Ecology 54: 427-432.

Holt, R. D. 2002. Food webs in space: on the interplay of dynamic instability and spatial processes. - Ecol. Res. 17: 261-273.

Hubbell, S. 2001. The unified neutral theory of biodiversity and biogeography. - Princeton Univ. Press.

Hubbell, S. P. 2005. The neutral theory of biodiversity and biogeography and Stephen Jay Gould. - Paleobiology 31: $122-132$.

Keitt, T. H. et al. 1997. Detecting critical scales in fragmented landscapes. - Conserv. Ecol. <www.consecol.org/> 1: 4 (online).

Kingman, J. F. C. 1982. On the genealogy of large populations. - J. Appl. Prob. 19: 27-43.

Leibold, M. and McPeek, M. 2006. Coexistence of the niche and neutral perspectives in community ecology. - Ecology 87: 1399-1410.

Leibold, M. et al. 2004. The metacommunity concept: a framework for multi-scale community ecology. - Ecol. Lett. 7: 601-613.

Levin, S. 1992. The problem of pattern and scale in ecology: the Robert H. MacArthur award lecture. - Ecology 73: 1943-1967.

MacArthur, R. H. and Wilson, E. O. 1967. The theory of island biogeography. - Princeton Univ. Press.

McGill, B. J. et al. 2006. Empirical evaluation of neutral theory. Ecology 87: 1411-1423.

McRae, B. H. 2006. Isolation by resistance. - Evolution 60: 1551-1561.

Minor, E. and Urban, D. 2008. A graph-theory framework for evaluating landscape connectivity and conservation planning. Conserv. Biol. 22: 297-307.

Mouillot, D. and Gaston, K. 2007. Geographical range size heritability: what do neutral models with different modes of speciation predict? - Global Ecol. Biogeogr. 16: 367-380.

Muneepeerakul, R. et al. 2007. A neutral metapopulation model of biodiversity in river networks. - J. Theor. Biol. 245: 351-363.

Muneepeerakul, R. et al. 2008. Neutral metacommunity models predict fish diversity patterns in Mississippi-Missouri basin. Nature 453: 220-222.

Newman, M. E. 2003. The structure and function of complex networks. - SIAM Rev. 45: 167-256.

Olesen, J. et al. 2007. The modularity of pollination networks. Proc. Natl Acad. Sci. USA 104: 19891-19896.

Rangel, T. and Diniz-Filho, J. 2005. Neutral community dynamics, the mid-domain effect and spatial patterns in species richness. Ecol. Lett. 8: 783-790.

Ricklefs, R. 1987. Community diversity: relative roles of local and regional processes. - Science 235: 167-171.

Rosenzweig, M. 1995. Species diversity in space and time. Cambridge Univ. Press.

Rosindell, J. and Cornell, S. J. 2007. Species-area relationships from a spatially explicit neutral model in an infinite landscape. Ecol. Lett. 10: 586-595.

Rosindell, J. et al. 2008. A coalescence approach to spatial neutral ecology. - Ecol. Inf. 3: 259-271.

Scott, J. 2000. Social network analysis: a handbook. - Sage.

Simpson, E. 1949. Measurement of diversity. - Nature 163: 688.

Simpson, G. 1964. Species density of North American recent mammals. - Syst. Zool. 13: 57-73. 
Spector, S. 2002. Biogeographic crossroads as priority areas for biodiversity conservation. - Conserv. Biol. 16: 1480-1487.

Strogatz, S. 2001. Exploring complex networks. - Nature 410: 268-276.

Urban, D. and Keitt, T. 2001. Landscape connectivity: a graphtheoretic perspective. - Ecology 82: 1205-1218.

Supplementary material (available online as Appendix O18272 at www.oikos.ekol.lu.se/appendix). Appendix 1: Fig. S1a-d and Fig. S2a-g.
Urban, D. et al. 2009. Graph models of habitat mosaics. - Ecol. Lett. 12: 260-273.

Wasserman, S. and Faust, K. 1994. Social network analysis: methods and applications. - Cambridge Univ. Press.

Wilkins, J. and Wakeley, J. 2002. The coalescent in a continuous, finite, linear population. - Genetics 161: 873-888. 